\title{
Polynomial Moment Relaxation for MIMO Detection
}

\author{
Tao Cui, Tracey Ho \\ Department of Electrical Engineering \\ California Institute of Technology \\ Pasadena, CA, USA 91125 \\ Email: $\{$ taocui, tho\}@caltech.edu
}

\author{
Chintha Tellambura \\ Department of Electrical and Computer Engineering \\ University of Alberta \\ Edmonton, AB, Canada T6G 2V4 \\ Email: chintha@ece.ualberta.ca
}

\begin{abstract}
We develop a polynomial-time detector for maximum likelihood (ML) detection over multiple-input multipleoutput (MIMO) channels. Our proposed polynomial moment relaxation (PMR) detection gives a unified framework for MIMO detection with relaxation including semi-definite relaxation as a special case. We give three approaches to replace a finite alphabet constraint with a polynomial constraint. Since both the objective function and the constraints are polynomials, we use a moment relaxation approach by applying the dual theories of moments and positive polynomials solvable by semi-definite programming. With different relaxation orders, our PMR achieve a flexible trade-off between complexity and performance.
\end{abstract}

\section{INTRODUCTION}

Multiple-input multiple-output (MIMO) systems, employing multiple antennas at both the transmitter and the receiver, can potentially achieve remarkably high spectral efficiencies in rich scattering multipath environments. As a result, the design of MIMO communication systems for high date-rate wireless communications has attracted a significant interest. A prime example is the Bell Laboratories layered space time (BLAST) architecture [1], which has been proposed to exploit the potentially enormous MIMO link capacity.

In spatial multiplexing systems such as BLAST, a fundamental receiver function is the detection of transmitted data symbols. The optimal maximum likelihood (ML) detector achieves the minimum error probability for i.i.d. data symbols, a requirement which holds in many cases. However, the complexity of the ML detector (MLD) grows exponentially in the number of transmit antennas and the signal constellation size, making it computationally prohibitive in most cases. Therefore, various computationally efficient linear receivers such as the zero-forcing (ZF) detector and the minimum meansquare error (MMSE) detector are popular. In [1], the VBLAST detector with optimal ordering, nulling and interference cancellation is proposed. However, these suboptimal detectors cannot fully exploit the diversity order offered by the MIMO channel. In [2], sphere decoding (SD) for the MLD is proposed for attaining low complexity in high SNR. Even though the VLSI implementations of SD have already been reported in [3], the variation of its time complexity can be high, leading to undesirable variable detection delays. Alternative detectors with constant time complexity are desirable.

In this paper, we consider a relaxation approach to MIMO detection which requires minimizing a quadratic cost function over the discrete set of all possible transmit vectors. In the relaxation approach, this discrete set is embedded in a larger multidimensional continuous space and the minimization is performed over this continuous space. The resulting minimum solution is mapped back into the original discrete space. In [4], semi-definite relaxation (SDR) has been applied to codedivision multiple-access (CDMA) systems with binary phase shift keying (BPSK). However, SDR can be loose and is not applicable to any constellations, which motivates the search for tighter and universal relaxations. We present a unified framework for MIMO detection with relaxation including SDR as a special case, and some existing linear suboptimal detectors can be considered to be further relaxations of the polynomial moment relaxation (PMR). We find that any finite-alphabet constraint can be relaxed to be a polynomial constraint. Three approaches for the formulation of the polynomial constraint are given, resulting in different tightness of relaxation. Since both the objective function and the constraints are polynomials, we use a moment relaxation approach by using the dual theories of moments and positive polynomials [5], [6] solvable by semi-definite programming (SDP) [7] with a polynomial-time complexity. As the relaxation order increases, the solution of the relaxed problem converges to that of the MLD. Under certain conditions, the optimal solution is achieved by the PMR. Our PMR provides a flexible trade-off between complexity and performance. By fixing the number of iterations in SDP, we ensure that the PMR has constant average time complexity.

Notation: Bold symbols denote matrices or vectors. $(\cdot)^{T}$ denotes transpose. $\mathbb{R}$ denotes the real number set. $\Re\{x\}$ and $\Im\{x\}$ denote the real part and image part of $x$, respectively. $\|(\cdot)\|^{2}$ is 2-norm of (.). A circularly complex Gaussian variable with mean $\mu$ and variance $\sigma^{2}$ is denoted by $z \sim \mathcal{C N}\left(\mu, \sigma^{2}\right)$. For a matrix $\mathbf{A}, \mathbf{A} \succeq 0$ denotes positive semi-definiteness. The operator $\otimes$ denotes Kronecker product. $\jmath=\sqrt{-1}$. We use Matlab notation throughout this paper.

\section{SYSTEM MODEL}

A MIMO system with $N_{T}$ transmit antennas and $N_{R}$ receive antennas is considered. We focus on spatial multiplexing systems, where the signals are spatially independent rather than jointly encoded. $N_{T} k$ binary bits are mapped into $N_{T}$ complex symbols from a finite constellation $\mathcal{Q}$ of size $s=2^{k}$ and $\mathcal{Q}=\left\{q_{1}, q_{2}, \ldots, q_{s}\right\}$. The data stream is demultiplexed into $N_{T}$ equal length substreams, and each of which is 
simultaneously sent through $N_{T}$ antennas over a rich scattering channel. We assume that the MIMO channel is flat fading. Each receive antenna receives signals from all the $N_{T}$ transmit antennas. The received signals can be written as

$$
\mathbf{r}=\mathbf{H x}+\mathbf{n}
$$

where $\mathbf{x}=\left[x_{1}, \ldots, x_{N_{T}}\right]^{T}, x_{i} \in \mathcal{Q}$, is the transmitted signal vector, $\mathbf{r}=\left[r_{1}, \ldots, r_{N_{R}}\right]^{T}, r_{i} \in \mathcal{C}$, is the received signal vector, $\mathbf{H}=\left[h_{i, j}\right] \in \mathcal{C}^{N_{R} \times N_{T}}$ is the channel matrix, and $\mathbf{n}=\left[n_{1}, \ldots, n_{N_{R}}\right]^{T}, n_{i} \sim \mathcal{C N}\left(0, \sigma_{n}^{2}\right)$, is an additive white Gaussian noise (AWGN) vector. The elements of $\mathbf{H}$ are identically independent distributed (i.i.d.) complex Gaussian, $h_{i, j} \sim \mathcal{C N}(0,1)$. The components of $\mathbf{n}$ are i.i.d. and $n_{i} \sim$ $\mathcal{C N}\left(0, \sigma_{n}^{2}\right)$. We assume that the channel is perfectly known to the receiver, but we do not assume $N_{T} \leq N_{R}$. The detection proposed in this paper can also handle the case with $N_{T}>N_{R}$. Note (1) models any linear, synchronous and flat fading channels, i.e., it can be directly applied to multiuser detection in CDMA. Therefore, the detector proposed in this paper can be readily applied to CDMA systems.

Assuming uncorrelated noise and transmitted signals, the MLD that minimizes the average error probability is given by

$$
\hat{\mathbf{x}}=\underset{\mathbf{x} \in \mathcal{Q}^{N_{T}}}{\arg \min }\|\mathbf{r}-\mathbf{H} \mathbf{x}\|^{2} .
$$

Due to the discrete nature of $\mathcal{Q},(2)$ is a NP-hard problem and exhaustive search for $\hat{\mathbf{x}}$ has a complexity exponential in $N_{T}$.

\section{Polynomial CONSTRAint FORMUlation}

Three approaches for the relaxation of a finite alphabet constraint to a polynomial constraint are given in this section.

\section{A. Formulation I}

We find that each $x_{i} \in \mathcal{Q}$ can be expressed as

$$
x_{i}=f_{1}\left(\mathbf{b}^{i}\right)+\jmath f_{2}\left(\mathbf{b}^{i}\right), i=1, \ldots, s
$$

where $\mathbf{b}^{i}=\left[b_{1}^{i}, \ldots, b_{k}^{i}\right]^{T}, b_{j}^{i} \in[-1,1]$ for $j=1, \ldots, k$, and $f_{1}\left(\mathbf{b}^{i}\right), f_{2}\left(\mathbf{b}^{i}\right)$ are polynomials in $\mathbf{b}^{i}$. Therefore, Eq. (2) becomes

$$
\hat{\tilde{\mathbf{b}}}=\underset{\tilde{\mathbf{b}} \in\{-1,1\}^{N_{T} k}}{\arg \min }\left\|\mathbf{r}-\mathbf{H}\left(\mathbf{f}_{1}(\tilde{\mathbf{b}})+\jmath \mathbf{f}_{2}(\tilde{\mathbf{b}})\right)\right\|^{2}
$$

where $\tilde{\mathbf{b}}=\left[\left(\mathbf{b}^{1}\right)^{T}, \ldots,\left(\mathbf{b}^{N_{T}}\right)^{T}\right]^{T}$ and $\mathbf{f}_{1}(\tilde{\mathbf{b}})=$ $\left[f_{1}\left(\mathbf{b}^{1}\right), \ldots, f_{1}\left(\mathbf{b}^{N_{T}}\right)\right]^{T}$. Eq. (6) can be further relaxed as

$$
\begin{gathered}
\hat{\tilde{\mathbf{b}}}=\underset{\tilde{\mathbf{b}} \in \mathbb{R}^{N_{T}}}{\arg \min }\left\|\mathbf{r}-\mathbf{H}\left(\mathbf{f}_{1}(\tilde{\mathbf{b}})+\jmath \mathbf{f}_{2}(\tilde{\mathbf{b}})\right)\right\|^{2} \\
\text { s.t. } \tilde{b}_{i}^{2}=1, i=1, \ldots, N_{T} k .
\end{gathered}
$$

For example, for QPSK, $\mathcal{Q}=\{1+\jmath, 1-\jmath,-1+\jmath,-1-\jmath\}$, we have

$$
f_{1}\left(b_{1}, b_{2}\right)=b_{1}, f_{2}\left(b_{1}, b_{2}\right)=b_{2} .
$$

For 8-QAM, $\mathcal{Q}=\{1+\jmath, 1-\jmath,-1+\jmath,-1-\jmath, 3+\jmath, 3-$ $\jmath,-3+\jmath,-3-\jmath\}$, and

$$
f_{1}\left(b_{1}, b_{2}, b_{3}\right)=b_{1}+2 b_{2}, f_{2}\left(b_{1}, b_{2}, b_{3}\right)=b_{3} \text {. }
$$

For 8-PSK, $\mathcal{Q}=\left\{e^{\jmath \pi i / 4} \mid i=0,1, \ldots, 7\right\}$, and

$$
\begin{aligned}
& f_{1}\left(b_{1}, b_{2}, b_{3}\right)=\frac{1-\sqrt{2}}{4} b_{1} b_{3}-\frac{1}{4} b_{2} b_{3}+\frac{1+\sqrt{2}}{4} b_{1}-\frac{1}{4} b_{2} \\
& f_{2}\left(b_{1}, b_{2}, b_{3}\right)=\frac{1-\sqrt{2}}{4} b_{2} b_{3}+\frac{1}{4} b_{1} b_{3}+\frac{1+\sqrt{2}}{4} b_{2}+\frac{1}{4} b_{1} .
\end{aligned}
$$

For squared QAM with $s=2^{2 k_{1}}$, we have

$$
\begin{aligned}
f_{1}\left(b_{1}, \ldots, b_{k_{1}}\right) & =\sum_{i=1}^{k_{1}} b_{i} 2^{i-1} \\
f_{2}\left(b_{k_{1}+1}, \ldots, b_{2 k_{1}}\right) & =\sum_{i=1}^{k_{1}} b_{i+k_{1}} 2^{i-1} .
\end{aligned}
$$

\section{B. Formulation II}

Due to the finite alphabet nature of $\mathcal{Q}$, each $x_{i} \in \mathcal{Q}$ satisfies following equation

$$
f\left(x_{i}\right)=\prod_{j=1}^{s}\left(x_{i}-q_{j}\right)=0 .
$$

The ML detection problem (2) can thus be relaxed as

$$
\begin{aligned}
\hat{\mathbf{x}}=\underset{\mathbf{x} \in \mathcal{C}^{N_{T}}}{\arg \min }\|\mathbf{r}-\mathbf{H} \mathbf{x}\|^{2} \\
\quad \text { s.t. } f\left(x_{i}\right)=\prod_{j=1}^{s}\left(x_{i}-q_{j}\right)=0, i=1, \ldots, N_{T} .
\end{aligned}
$$

To avoid the complex operation, Eq. (11) can be transformed into a real problem as

$$
\begin{aligned}
& \hat{\tilde{\mathbf{x}}}=\underset{\tilde{\mathbf{x}} \in \mathbb{R}^{2 N_{T}}}{\arg \min }\|\tilde{\mathbf{r}}-\tilde{\mathbf{H}} \tilde{\mathbf{x}}\|^{2} \\
& \text { s.t. } f_{1}\left(\Re\left\{x_{i}\right\}, \Im\left\{x_{i}\right\}\right)=0 \text { and } f_{2}\left(\Re\left\{x_{i}\right\}, \Im\left\{x_{i}\right\}\right)=0 \\
& \quad i=1, \ldots, N_{T}
\end{aligned}
$$

where

$$
\tilde{\mathbf{r}}=\left[\begin{array}{c}
\Re\{\mathbf{r}\} \\
\Im\{\mathbf{r}\}
\end{array}\right], \tilde{\mathbf{x}}=\left[\begin{array}{c}
\Re\{\mathbf{x}\} \\
\Im\{\mathbf{x}\}
\end{array}\right]
$$

and

$$
\tilde{\mathbf{H}}=\left[\begin{array}{cc}
\Re\{\mathbf{H}\} & -\Im\{\mathbf{H}\} \\
\Im\{\mathbf{H}\} & \Re\{\mathbf{H}\}
\end{array}\right] .
$$

$f_{1}\left(\Re\left\{x_{i}\right\}, \Im\left\{x_{i}\right\}\right)=\Re\left\{f\left(x_{i}\right)\right\}$ and $f_{2}\left(\Re\left\{x_{i}\right\}, \Im\left\{x_{i}\right\}\right)=$ $\Im\left\{f\left(x_{i}\right)\right\}$, which are also polynomial in $\Re\left\{x_{i}\right\}$ and $\Im\left\{x_{i}\right\}$ with maximum order $k$. For example, for BPSK, $f_{1}\left(\Re\left\{x_{i}\right\}, \Im\left\{x_{i}\right\}\right)=\Re\left\{x_{i}\right\}^{2}-1$ and $f_{2}\left(\Re\left\{x_{i}\right\}, \Im\left\{x_{i}\right\}\right)=0$.

Specifically, for decouplable constellations, i.e., squared QAM, $f_{1}\left(\Re\left\{x_{i}\right\}, \Im\left\{x_{i}\right\}\right)=f\left(\Re\left\{x_{i}\right\}\right), f_{2}\left(\Re\left\{x_{i}\right\}, \Im\left\{x_{i}\right\}\right)=$ $f\left(\Im\left\{x_{i}\right\}\right)$, and $f_{1}(\cdot), f_{2}(\cdot)$ have the same form. Therefore, (12) can be simplified as

$$
\begin{aligned}
\hat{\tilde{\mathbf{x}}}=\underset{\tilde{\mathbf{x}} \in \mathbb{R}^{2 N_{T}}}{\arg \min }\|\tilde{\mathbf{r}}-\tilde{\mathbf{H}} \tilde{\mathbf{x}}\|^{2} & \\
& \text { s.t. } f\left(\tilde{x}_{i}\right)=0, i=1, \ldots, 2 N_{T}
\end{aligned}
$$

where $\tilde{x}_{i}$ is the $i$-th element of $\tilde{\mathbf{x}}$. For example, for 16-QAM, $f\left(\tilde{x}_{i}\right)=\left(\tilde{x}_{i}+3\right)\left(\tilde{x}_{i}-3\right)\left(\tilde{x}_{i}+1\right)\left(\tilde{x}_{i}-1\right)=\tilde{x}_{i}^{4}-10 \tilde{x}_{i}^{2}+9$. 


\section{Formulation III}

Due to the finite alphabet nature of $\mathcal{Q}$, each $x_{i} \in \mathcal{Q}$ also satisfies following equation

$$
x_{i}=f\left(\mathbf{b}^{i}\right)=b_{1}^{i} q_{1}+, \ldots,+b_{s}^{i} q_{s}=\mathbf{q}^{T} \mathbf{b}^{i} \text { and } \sum_{j=1}^{s} b_{j}^{i}=1,
$$

where $\mathbf{b}^{i}=\left[b_{1}^{i}, \ldots, b_{s}^{i}\right]^{T}, b_{j}^{i} \in\{0,1\}$, and $\mathbf{q}=\left[q_{1}, \ldots, q_{s}\right]^{T}$. Eq. (2) can be written as

$$
\begin{aligned}
\hat{\tilde{\mathbf{b}}}=\underset{\tilde{\mathbf{b}} \in\{0,1\}^{N_{T} s}}{\arg \min }\|\mathbf{r}-\mathbf{H A} \tilde{\mathbf{b}}\|^{2} \\
\text { s.t. } \mathbf{B} \mathbf{b}=\mathbf{e}_{N_{T}}
\end{aligned}
$$

where $\tilde{\mathbf{b}}=\left[\left(\mathbf{b}^{1}\right)^{T}, \ldots,\left(\mathbf{b}^{N_{T}}\right)^{T}\right]^{T}, \mathbf{A}=\mathbf{I}_{N_{T}} \otimes \mathbf{q}^{T}, \mathbf{B}=$ $\mathbf{I}_{N_{T}} \otimes \mathbf{e}_{s}^{T}$, and $\mathbf{e}_{n}$ denotes the $n \times 1$ vector of all ones. By removing the finite alphabet on $\{0,1\}$, Eq. (17) can be further relaxed as

$$
\begin{aligned}
\hat{\tilde{\mathbf{b}}}=\underset{\tilde{\mathbf{b}} \in \mathbb{R}^{N_{T} s}}{\arg \min }\|\mathbf{r}-\mathbf{H A} \tilde{\mathbf{b}}\|^{2} & \\
& \text { s.t. } \mathbf{B} \tilde{\mathbf{b}}=\mathbf{e}_{N_{T}}, \quad \tilde{b}_{i}^{2}-\tilde{b}_{i}=0, i=1, \ldots, N_{T} s
\end{aligned}
$$

where $\tilde{b}_{i}$ is the $i$-th entry of $\tilde{\mathbf{b}}$.

Note that (17) can be readily transformed into an optimization problem on $\{-1,1\}$ by replace $\tilde{b}_{i}$ with $\left(\tilde{b}_{i}^{\prime}+1\right) / 2$, $\tilde{b}_{i}^{\prime} \in\{-1,1\}$.

\section{Remarks:}

- After relaxation, (5) and (18) have $N_{T} k$ and $N_{T} s$ variables, respectively. If $N_{T} k>N_{R}$ or $N_{T} s>N_{R}$, (5) or (18) becomes a rank deficient system, which can be solved using the moment relaxation in Section IV. However, increasing the rank difference $N_{T} k-N_{R}$ or $N_{T} s-N_{R}$, the performance may degrade significantly.

- The polynomial order in the formulation (15) is $s / 2$ for squared QAM constellations. Increasing the constellation size, the problem size increases drastically in the moment relaxation in Section IV. To overcome these difficulties, hybrid formulations can be used. For example, for 16QAM, we can combine formulations I and II, and for each $x_{i} \in \mathcal{Q}$, we have $x_{i}=b^{i} \tilde{x}_{i}$, where $b^{i} \in\{-1,1\}$ and $\tilde{x}_{i}^{2}-4 \tilde{x}_{i}+3=0$. The polynomial order is reduced from 4 to 3 . We can also combine formulations I and III. For each $x_{i} \in \mathcal{Q}$, we have $x_{i}=b_{1}^{i}\left(3 b_{2}^{i}+b_{3}^{i}\right)$, where $b_{1}^{i} \in\{-1,1\}, b_{2}^{i}, b_{3}^{i} \in\{0,1\}$ and $b_{2}^{i}+b_{3}^{i}=1$. The number of variables is reduced from 4 to 3 .

\section{Moment Relaxation FOR POLYNOMials}

Both the objective function and constraints in the three formulations are polynomials. In the following, we consider a general polynomial constrained optimization problem:

$$
\begin{array}{ll}
\mathbb{P}: & \min _{\mathbf{x} \in \mathbb{R}^{n}} p(\mathbf{x}) \\
& \text { s.t. } \quad g_{i}(\mathbf{x}) \geq 0, i=1, \ldots, r,
\end{array}
$$

where $p(\mathbf{x})$ is a real-valued polynomial of degree at most $m$, and $g_{i}(\mathbf{x})$ is a real-valued polynomial of degree at most $w_{i}$, $i=1, \ldots, r$. If there exists an equality constraint $f(\mathbf{x})=0$, we can rewrite it as $f(\mathbf{x}) \geq 0$ and $-f(\mathbf{x}) \geq 0$. $\mathbb{P}$ includes all the three polynomial formulations in Section III. $\mathbb{P}$ can be written as

$$
\mathbb{P}_{K}: \quad \min _{\mathbf{x} \in \mathcal{K}} p(\mathbf{x})
$$

where $\mathcal{K}$ is a compact set defined by polynomial inequalities $g_{i}(\mathbf{x}) \geq 0$.

Before preceding, we define the basis for the $m$-degree realvalued polynomials as

$$
1, x_{1}, \ldots, x_{n}, x_{1}^{2}, x_{1} x_{2}, \ldots, x_{n}^{2}, \ldots, x_{1}^{m}, \ldots, x_{n}^{m} .
$$

Let $s(m)$ be its dimension. The $m$-degree polynomial $p(\mathbf{x})$ can then be written as

$$
p(\mathbf{x})=\sum_{\alpha} p_{\alpha} \mathbf{x}^{\alpha}
$$

where $\alpha=\left[\alpha_{1}, \ldots, \alpha_{n}\right], \mathbf{x}^{\alpha}=x_{1}^{\alpha_{1}} x_{2}^{\alpha_{2}} \cdots x_{n}^{\alpha_{n}}$, and $\sum_{i=1}^{n} \alpha_{i} \leq m . \mathbf{p}=\left[p_{[0,0, \ldots, 0]}, \ldots, p_{[0,0, \ldots, m]}\right]^{T} \in \mathbb{R}^{s(m)}$ is the coefficient vector of $p(\mathbf{x})$ in the basis (21).

The mean idea of moment relaxation is to replace $\mathbb{P}_{K}$ with the equivalent problem [5]

$$
\mathcal{P}_{K}: \quad \min _{\mu \in \mathcal{P}(\mathcal{K})} \int p(\mathbf{x}) \mu(d \mathbf{x})
$$

where $\mathcal{P}_{K}$ is the space of finite measures on $\mathcal{K}$. We have the following proposition

Proposition 1: The problems $\mathbb{P}_{K}$ and $\mathcal{P}_{K}$ are equivalent in that

(a) $\inf \mathbb{P}_{K}=\inf \mathcal{P}_{K}$.

(b) If $\mathbf{x}^{*}$ is a global minimizer of $\mathcal{P}_{K}, \mu=\delta_{\mathbf{x}^{*}}$ is a global minimizer of $\mathcal{P}_{K}$.

(c) If $\mathrm{x}^{*}$ is the unique global minimizer of $\mathcal{P}_{K}, \mu=\delta_{\mathrm{x}^{*}}$ is the unique global minimizer of $\mathcal{P}_{K}$.

The proof of Proposition 1 is trivial and can be found in [5].

Since $p(\mathbf{x})$ is a polynomial of degree at most $m$, $\int p(\mathbf{x}) \mu(d \mathbf{x})$ involves only the moments of $\mu$ up to order $m$, which can be expressed as

$$
\int p(\mathbf{x}) \mu(d \mathbf{x})=\sum_{\alpha} p_{\alpha} \int \mathbf{x}^{\alpha} \mu(d \mathbf{x})=\sum_{\alpha} p_{\alpha} y_{\alpha}
$$

where $y_{\alpha}=\int \mathbf{x}^{\alpha} \mu(d \mathbf{x})$. Clearly, the objective function in $\mathcal{P}_{K}$ is linear in the moment variables $y_{\alpha}$. One may promptly conclude that non-convex problem $\mathbb{P}_{K}$ is transformed into a linear programming problem or a convex problem in $\mathbf{y}=$ $\left[y_{[0, \ldots, 0]}, \ldots, y_{[0, \ldots, m]}\right]^{T}$, which can be readily solved. However, $\mathbf{y}$ is also constrained to be the moments of a representing measure $\mu$. Given an arbitrary $\mathbf{y}$, there may not exist a measure $\mu$, whose moments up to order $m$ coincide with $\mathbf{y}$.

In the one-dimensional case $n=1$, characterizing those $\mathbf{y}$ that have a representing measure is called the Hamburger moment problem [8]. Various necessary and sufficient conditions for the existence of a representing measure are available in the one-dimensional case. The theory of moments is in duality with the theory of nonnegative polynomials and Hilbert's 17th problem on the representation of nonnegative polynomials 
as sum of squares, which is always possible in the onedimensional case. However, in the multidimensional case, not every nonnegative polynomial can be written as a sum of squares [5], and no sufficient condition exists for the existence of a representing measure. We give a necessary condition in the following.

We define the moment matrix $M_{m}(\mathbf{y})$ of dimension $s(m)$. $M_{m}(\mathbf{y})$ is constructed as follows. If $M_{m}(\mathbf{y})(1, i)=y_{\alpha}$ and $M_{m}(\mathbf{y})(j, 1)=y_{\beta}$, then $M_{m}(\mathbf{y})(i, j)=y_{\alpha+\beta}$, where $\alpha+\beta=\left[\alpha_{1}+\beta_{1}, \ldots, \alpha_{n}+\beta_{n}\right]$. Let $q(\mathbf{x})$ be an arbitrary polynomial of degree at most $m$ with the coefficient vector $\mathbf{q}=\left[q_{[0,0, \ldots, 0]}, \ldots, q_{[0,0, \ldots, m]}\right]^{T}$. We have

$$
\int q^{2}(\mathbf{x}) \mu(d \mathbf{x})=\mathbf{q}^{T} M_{m}(\mathbf{y}) \mathbf{q} \geq 0
$$

for all $\mathbf{q} \in \mathbb{R}^{m}$. Therefore, the necessary condition that $\mathbf{y}$ corresponds to moments of some probability measure $\mu$ on $\mathcal{K}$ is that $M_{m}(\mathbf{y})$ must be positive semi-definite or $M_{m}(\mathbf{y}) \succeq 0$. Furthermore, since $g_{i}(\mathbf{x}) \geq 0$, we have

$$
\int g_{i}(\mathbf{x}) q^{2}(\mathbf{x}) \mu(d \mathbf{x})=\mathbf{q}^{T} M_{m}\left(\mathbf{g}_{i} \mathbf{y}\right) \mathbf{q} \geq 0
$$

for all $\mathbf{q} \in \mathbb{R}^{m}$, where the $(j, k)$-th entry of $M_{m}\left(\mathbf{g}_{i} \mathbf{y}\right)$ is defined as

$$
M_{m}\left(\mathbf{g}_{i} \mathbf{y}\right)(j, k)=\sum_{\alpha}\left(g_{i}\right)_{\alpha} y_{\alpha+\beta},
$$

and $y_{\beta}$ is the $(j, k)$-th entry of $M_{m}(\mathbf{y})$. Thus, we also require $M_{m}\left(\mathbf{g}_{i} \mathbf{y}\right) \succeq 0$.

Let $\tilde{w}_{i}=\left\lceil w_{i} / 2\right\rceil$ be the smallest integer larger than $w_{i} / 2$, and choose an integer $N \geq\lceil m / 2\rceil$ and $N \geq \max _{i} w_{i}$. Since $p(\mathbf{x})$ is also a polynomial of degree at most $2 N$ with the coefficients of monomials of degree higher than $m$ zero, we also have $M_{N}(\mathbf{y}) \succeq 0$, and similarly $M_{N-\tilde{w}_{i}}\left(\mathbf{g}_{i} \mathbf{y}\right) \succeq 0$. The $N$-th order moment relaxation problem is given by the following positive semi-definite programming problem

$$
\begin{aligned}
\mathbb{Q}_{K}^{N}: \quad \min _{\mathbf{y}} & \mathbf{p}^{T} \mathbf{y} \\
\text { s.t. } & M_{N}(\mathbf{y}) \succeq 0 \\
& M_{N-\tilde{w}_{i}}\left(\mathbf{g}_{i} \mathbf{y}\right) \succeq 0, \quad i=1, \ldots, r .
\end{aligned}
$$

Note that $\mathbb{Q}_{K}^{N}$ is a convex optimization problem since its object and constraint are both convex. Let $M_{N}(\mathbf{y})=\mathbf{B}_{0}+$ $\sum_{\alpha \neq \mathbf{0}} \mathbf{B}_{\alpha} y_{\alpha}\left(\mathbf{y}_{[0, \ldots, 0]}=1\right)$ and $M_{N-\tilde{w}_{i}}\left(\mathbf{g}_{i} \mathbf{y}\right)=\sum_{\alpha} \mathbf{C}_{i, \alpha} y_{\alpha}$. From the duality theory of SDP [7], the dual problem of $\mathbb{Q}_{K}^{N}$ is defined by

$$
\begin{gathered}
\left(\mathbb{Q}_{K}^{N}\right)^{*}: \max _{\mathbf{X}, \mathbf{Z}_{i}}-\mathbf{X}(1,1)-g_{i}(\mathbf{0}) \mathbf{Z}_{i}(1,1) \\
\text { s.t. } \operatorname{tr}\left(\mathbf{X}, \mathbf{B}_{\alpha}\right)+\sum_{i=1}^{r} \operatorname{tr}\left(\mathbf{Z}_{i}, \mathbf{C}_{i, \alpha}\right)=p_{\alpha}, \alpha \neq \mathbf{0} \\
\quad \mathbf{X} \succeq 0, \mathbf{Z}_{i} \succeq 0, i=1, \ldots, r .
\end{gathered}
$$

Let $p_{K}^{*}$ and $\left(q_{K}^{N}\right)^{*}$ be the minimum value of $\mathbb{P}_{K}$ and $\mathbb{Q}_{K}^{N}$, respectively. We have the following theorem.

Theorem 1: [5] (a)

$$
\lim _{N \rightarrow+\infty}\left(q_{K}^{N}\right)^{*}=p_{K}^{*} .
$$

Moreover, as $N \rightarrow+\infty$, there is no duality gap between $\mathbb{Q}_{K}^{N}$ and $\left(\mathbb{Q}_{K}^{N}\right)^{*}$.

(b) If $p(\mathbf{x})-p_{K}^{*}$ has the representation

$$
p(\mathbf{x})-p_{K}^{*}=q(\mathbf{x})+\sum_{i=1}^{r} g_{i}(\mathbf{x}) t_{i}(\mathbf{x})
$$

where $q(\mathbf{x})$ is a polynomial of degree at most $2 N, t_{i}(\mathbf{x})$ is a polynomial of degree at most $2 N-w_{i}, i=1, \ldots, r$, all sums of squares, then $\left(q_{K}^{N}\right)^{*}=p_{K}^{*}$ and there is no duality gap between $\mathbb{Q}_{K}^{N}$ and $\left(\mathbb{Q}_{K}^{N}\right)^{*}$. For every optimal solution $\mathrm{x}^{*}$ of $\mathbb{P}_{K}$, the vector

$$
\mathbf{y}^{*}=\left[1, x_{1}^{*}, \ldots, x_{n}^{*},\left(x_{1}^{*}\right)^{2}, \ldots,\left(x_{1}^{*}\right)^{2 N}, \ldots,\left(x_{n}^{*}\right)^{2 N}\right]^{T}
$$

is a global minimizer of $\mathbb{Q}_{K}^{N}$. Moreover, for every optimal solution $\left(\mathbf{X}^{*}, \mathbf{Z}_{1}^{*}, \ldots, \mathbf{Z}_{r}^{*}\right)$ of $\left(\mathbb{Q}_{K}^{N}\right)^{*}$, we have

$$
p(\mathbf{x})-p_{K}^{*}=\sum_{i=1}^{r_{0}} \lambda_{i} q_{i}^{2}(\mathbf{x})+\sum_{i=1}^{r} g_{i}(\mathbf{x}) \sum_{j=1}^{r_{i}} \gamma_{i, j} t_{i, j}^{2}(\mathbf{x})
$$

where the coefficients vectors of the polynomials $q_{i}(\mathbf{x})$, $t_{i, j}(\mathbf{x})$ are the eigenvectors of $\mathbf{X}^{*}$ and $\mathbf{Z}_{i}^{*}$ with respective to eigenvalues $\lambda_{i}, \gamma_{i, j}$, and $r_{0}$ and $r_{i}$ is the number of positive eigenvalues of $\mathbf{X}^{*}$ and $\mathbf{Z}_{i}^{*}$, respectively.

The proof of Theorem 1 can be found in [5]. Theorem 1 implies that even though $M_{N}(\mathbf{y}) \succeq 0$ and $M_{N-\tilde{w}_{i}}\left(\mathbf{g}_{i} \mathbf{y}\right) \succeq$ 0 are only necessary conditions, they are also sufficient as $N \rightarrow+\infty$. Moreover, for finite $N,\left(q_{K}^{N}\right)^{*}$ always provides a lower bound on $p_{K}^{*}$.

In (5) and (18), the variables have $\{-1,+1\}$ or $\{0,1\}$ constraint. In (19), these constraints imply $x_{i}^{2}=x_{i}$ or $x_{i}^{2}=1$. Therefore, we have

$$
x_{1}^{\alpha_{1}} x_{2}^{\alpha_{2}} \cdots x_{n}^{\alpha_{n}}=x_{1}^{\beta_{1}} x_{2}^{\beta_{2}} \cdots x_{n}^{\beta_{n}},\left\{\begin{array}{cc}
\beta_{i}=0, & \alpha_{i}=0 \\
\beta_{i}=1, & \alpha_{i}>0
\end{array}\right.
$$

or

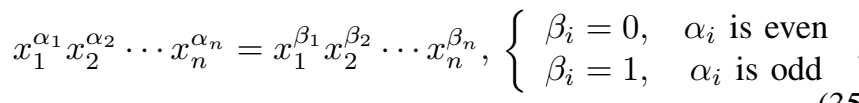

The variables $y_{\alpha}$ in $\mathbb{Q}_{K}^{N}$ can be replaced by $y_{\beta}$ in both the objective and constraint, and the constraints $\mathbb{Q}_{K}^{N}$ associated with $g_{i}(\mathbf{x})=x_{i}^{2}-x_{i}$ and $g_{i}(\mathbf{x})=x_{i}-x_{i}^{2}$ (for $\{0,1\}$ ) can be removed and are hidden in $y_{\beta}$. The problem $\mathbb{Q}_{K}^{N}$ can be simplified as

$$
\begin{aligned}
\mathbb{Q}_{K}^{N}: \quad \min _{\tilde{\mathbf{y}}} & \tilde{\mathbf{p}}^{T} \tilde{\mathbf{y}} \\
\text { s.t. } & \tilde{M}_{N}(\tilde{\mathbf{y}}) \succeq 0 \\
& \tilde{M}_{N-\tilde{w}_{i}}\left(\tilde{\mathbf{g}}_{i} \tilde{\mathbf{y}}\right) \succeq 0, \quad i=1, \ldots, \tilde{r}
\end{aligned}
$$

where $\tilde{\mathbf{p}}$ is formed by combining the coefficients of $y_{\alpha}$ with the same $y_{\beta}$ after transformation, $\tilde{r}$ is the number of constraints except those related to $\{0,1\}$ or $\{-1,1\}$, and $\tilde{\mathbf{y}}=\left[y_{[0, \ldots, 0]}, \ldots, y_{[1, \ldots, 1]}\right]^{T}$. Since the monomial $\mathbf{x}^{\beta}$ has a 
degree at most $n$ with $\beta=[1, \ldots, 1], \tilde{\mathbf{y}}$ has no more than $\sum_{i=1}^{n}\left(\begin{array}{c}n \\ i\end{array}\right)=2^{n}-1$ variables.

Let $\tilde{w}=\max _{i} \tilde{w}_{i}$. We have the following theorem for $\{0,1\}$ or $\{-1,+1\}$ constraint problem.

Theorem 2:

(a) For every $N \geq n+\tilde{w}$,

$$
\left(q_{K}^{N}\right)^{*}=p_{K}^{*} .
$$

There is no duality gap between $\mathbb{Q}_{K}^{N}$ and $\left(\mathbb{Q}_{K}^{N}\right)^{*}$.

(b) For every optimal solution $\mathbf{x}^{*}$ of $\mathbb{P}_{K}$, the vector

$$
\mathbf{y}^{*}=\left[1, x_{1}^{*}, \ldots, x_{n}^{*},\left(x_{1}^{*}\right)^{2}, \ldots,\left(x_{1}^{*}\right)^{2 N}, \ldots,\left(x_{n}^{*}\right)^{2 N}\right]^{T}
$$

is a global minimizer of $\mathbb{Q}_{K}^{N}$, and there exists a representing measure for $\mathbf{y}^{*}$.

The proof of Theorem 2 can be found in [6]. Since $\tilde{\mathbf{y}}$ has at most $2^{n}-1$ variables, it can be readily verified that when $N \geq n, \tilde{M}_{N}(\tilde{\mathbf{y}})$ has the same rank as $\tilde{M}_{n}(\tilde{\mathbf{y}})$, and $\tilde{M}_{N}(\tilde{\mathbf{y}}) \succeq 0$ is equivalent to $\tilde{M}_{n}(\tilde{\mathbf{y}}) \succeq 0$. Therefore, if $N \geq n+\tilde{w}$, (36) can be further simplified as

$$
\begin{aligned}
\mathbb{Q}_{K}^{N}: \quad \min _{\tilde{\mathbf{y}}} & \tilde{\mathbf{p}}^{T} \tilde{\mathbf{y}} \\
\text { s.t. } & \tilde{M}_{n}(\tilde{\mathbf{y}}) \succeq 0 \\
& \tilde{M}_{N-\tilde{w}_{i}}\left(\tilde{\mathbf{g}}_{i} \tilde{\mathbf{y}}\right) \succeq 0, \quad i=1, \ldots, \tilde{r} .
\end{aligned}
$$

Theorem 2 can also be understood from intuition. When $N \geq$ $n+w$, there are $2^{n}-1$ variables in $\mathbb{Q}_{K}^{N}$, which is equal to the number of candidates $\left(2^{n}\right)$ in the discrete search space minus one if no relaxation is used. In fact, $\mathbb{Q}_{K}^{N}$ does an exhaustive search, which should return the optimal solution. Generally, with our MIMO detection problem (5), (12) and (18) at hand, $\left(q_{K}^{N}\right)^{*}$ is guaranteed to achieve $p_{K}^{*}$ when the relaxation order $N$ is larger than a certain number $N^{\prime}$ from the same intuition.

$\mathbb{Q}_{K}^{N}$ is an SDP problem and can be efficiently solved using the Matlab toolbox SeDuMi [9] employing interior point method [7]. We can obtain $\mathbf{y}^{*}$ and $\left(\mathbf{X}^{*}, \mathbf{Z}_{1}^{*}, \ldots, \mathbf{Z}_{r}^{*}\right)$ by solving $\mathbb{Q}_{K}^{N}$. From Theorem 1 , if $\hat{\mathbf{x}}=\left[y_{[1,0, \ldots, 0]}^{*}, \ldots, y_{[0,0, \ldots, 1]}^{*}\right]^{T}$ is also the optimal solution of $\mathbb{P}_{K},\left(q_{K}^{N}\right)^{*}=p_{K}^{*}$. We decide whether $\hat{\mathbf{x}}$ achieves the global minimum by comparing $\left(q_{K}^{N}\right)^{*}$ with $p(\hat{\mathbf{x}})$. If $\left|\left(q_{K}^{N}\right)^{*}-p(\hat{\mathbf{x}})\right|<\epsilon|p(\hat{\mathbf{x}})|$, the global optimum is achieved and $\hat{\mathbf{x}}$ is the optimal solution, where $\epsilon$ is a threshold coefficient; otherwise, we can extract the optimal solution of $\mathbb{P}_{K}$ by exploiting the fact that if $\left(q_{K}^{N}\right)^{*}=p_{K}^{*}$, (33) holds. Since the right hand side of (33) is a sum of squares, a necessary and sufficient condition for $\mathbf{x}$ to achieve $p_{K}^{*}$ is $q_{i}(\mathbf{x})=0$, for $i=1, \ldots, r_{0}$. Therefore, the optimal solution is the common root of the set of equations $q_{i}(\mathbf{x})=0, i=1, \ldots, r_{0}$. The roots of a system of multivariate polynomial equations can be found by following the approach in [10], where the multivariate problem is reduced to a univariate problem. Due to the use of floating-point arithmetic, the set of equations may not have common roots. In [10], a clustering technique is used to approximate the common roots. If the clustering fails, it means that no common root can be found, $\left(q_{K}^{N}\right)^{*} \neq p_{K}^{*}$ and no optimal solution to $\mathbb{P}_{K}$ can be obtained by solving $\mathbb{Q}_{K}^{N}$. We can choose $\hat{\mathbf{x}}$ as a suboptimal solution.
The MIMO detector using the moment relaxation is called polynomial moment relaxation (PMR) detector.

\section{Remarks:}

- Our experimental results reveal that in practice, especially in MIMO detection, $\left(q_{K}^{N}\right)^{*}$ is very close to $p_{K}^{*}$ for a relatively small relaxation order $N$, i.e., $N=2$, 3 . In addition, the probability to achieve the exact optimal value $p_{K}^{*}$ is high for a small relaxation order. Therefore, our PMR detector performs close to the MLD.

- Let us consider the optimization problem

$$
\begin{gathered}
\min _{\mathbf{x} \in \mathbb{R}^{n}} \mathbf{x}^{T} \mathbf{A} \mathbf{x}-2 \mathbf{d}^{T} \mathbf{x} \\
\text { s.t. } x_{i} \in\{-1,1\} .
\end{gathered}
$$

When the moment relaxation order is $N=1$, the moment matrix $M_{1}(\mathbf{y})$ has dimension $n+1$. The $(i, j)$-th entry of $M_{1}(\mathbf{y})$ is

$$
M_{1}(\mathbf{y})(i, j)=\int x_{i-1} x_{j-1} \mu(d \mathbf{x})
$$

where $x_{0}=1$. Let $\mathbf{X}=M_{1}(\mathbf{y})$ and $\mathbb{Q}_{K}^{N}$ becomes

$$
\begin{aligned}
\mathbb{Q}_{K}^{N}: \quad \min _{\mathbf{X}} & \operatorname{tr}(\mathbf{Q X}) \\
\text { s.t. } & \mathbf{X} \succeq 0 \\
\quad & \mathbf{X}(i, i)=1, \quad i=1, \ldots, n
\end{aligned}
$$

where

$$
\mathbf{Q}=\left[\begin{array}{cc}
\mathbf{A} & -\mathbf{d} \\
-\mathbf{d} & 0
\end{array}\right]
$$

Eq. (42) is the same as the SDR for CDMA in [4]. Therefore, PMR generalizes SDR. It has been shown in [4] that some existing linear detectors can be considered to be further relaxations of the SDR. They are also further relaxations of the PMR.

In [4], randomization is applied after obtaining $\mathbf{X}$. The solution extraction procedure in PMR can also be applied to SDR. We first test whether $\left|\left(q_{K}^{N}\right)^{*}-p(\hat{\mathbf{x}})\right|<\epsilon|p(\hat{\mathbf{x}})|$. If so, $\hat{\mathbf{x}}$ is the optimal solution; otherwise, randomization is performed, which saves the complexity.

Since $M_{1}(\mathbf{y})$ is a submatrix of $M_{N}(\mathbf{y})$ for $N \geq 1$, we can get an approximate solution by performing randomization on $M_{1}(\mathbf{y})$ if the common roots finding fails and $\{-1,1\}$ constraint exists.

- For the PMR, the complexity per iteration is also $\mathcal{O}\left(s^{3}\right)$ [11], where $s$ is the number of variables in $\mathbb{Q}_{K}^{N}$. Given a tolerance $\epsilon$, the number of iterations required is at most $\mathcal{O}\left(s^{0.5} \log (1 / \epsilon)\right)$ [11]. A solution to $\mathbb{Q}_{K}^{N}$ can be found in at most $\mathcal{O}\left(s^{3.5}\right)$ operations. With relaxation order $N$, the number of variables for $\{-1,+1\}$ constraint is $s=$ $\sum_{i=1}^{2 N}\left(\begin{array}{c}n \\ i\end{array}\right)$, With small $N$ and large $n, s$ is significantly less than $2^{n}$. Moreover, the complexity of PMR can be made a constant by fixing the number of iterations in solving $\mathbb{Q}_{K}^{N}$. 


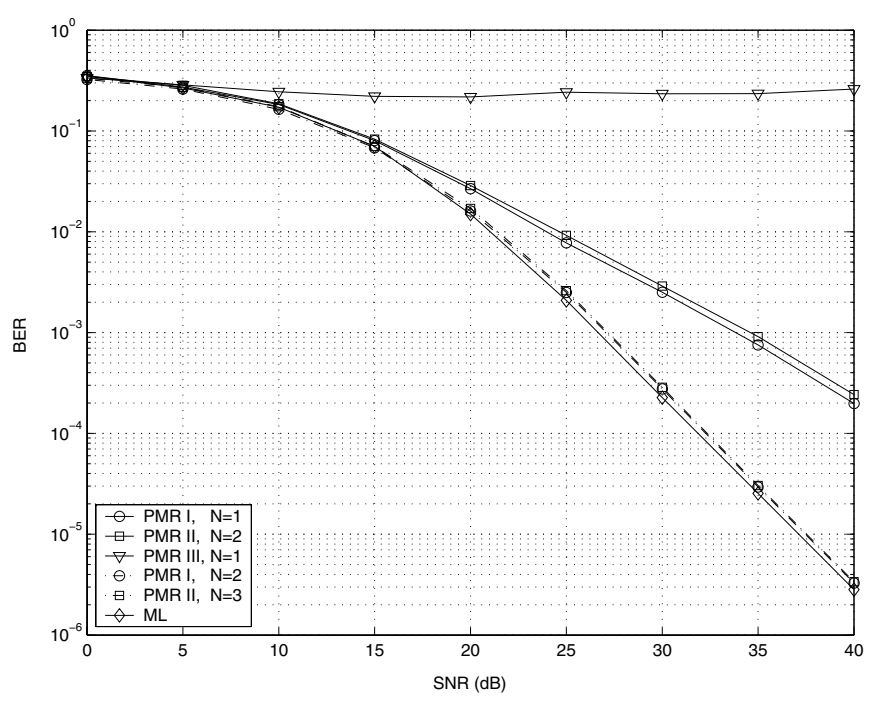

Fig. 1. Performance comparison of polynomial moment relaxation detectors in an $2 \times 2$ MIMO system with 16QAM.

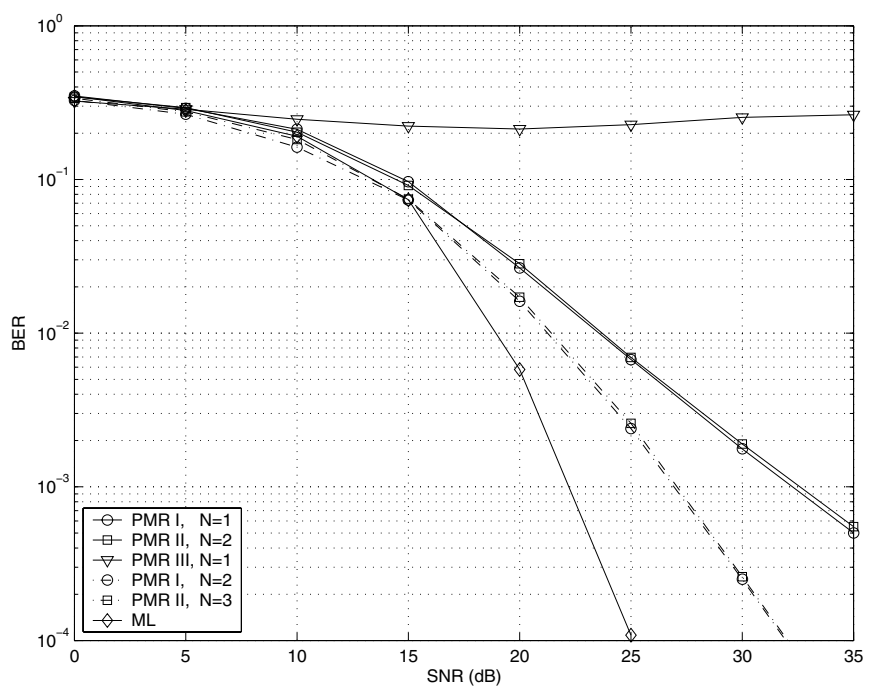

Fig. 2. Performance comparison of polynomial moment relaxation detectors in an $4 \times 4$ MIMO system with 16QAM.

\section{Simulation Results}

The error rates of our proposed polynomial moment relaxation detectors are simulated for a MIMO system over a flat Rayleigh fading channel. PMR I denotes the moment relaxation detector with formulation I. We compare our detectors with the MLD.

In Fig. 1, we compare different formulations for a $2 \times 2$ MIMO system with 16QAM. Both PMR I with $N=1$ and PMR II with $N=2$ can only achieve a diversity order 1 . An error floor is shown for PMR III. This is because the rank difference $N_{T} s-N_{R}$ is very large in Formulation III with $N=1$. A symbol error occurs if $\mathbf{H}$ is ill-conditioning. When $N$ increases by 1 , both PMR I and II perform close to the MLD and achieve a diversity order 2 .

Fig. 2 show the BER performance of different PMR detec- tors in a $4 \times 4$ MIMO system with 16QAM. Similarly, both PMR I with $N=1$ and PMR II with $N=2$ can only achieve a diversity order 1 , and an error floor is shown for PMR III. The number of variables for PMR I with $N=1$ and PMR II with $N=2$ is 289 and 494, respectively. Therefore, PMR I with $N=1$ has less complexity than PMR II with $N=2$, while the former performs slightly better than the latter. When $N$ is increased by one, the diversity order of both PMR I and II is also increased by one. Let $\tilde{w}=\max \tilde{w}_{i}$. We conjecture that the diversity order of PMR detector is $N-\tilde{w}+1$, which increases by increasing $N$.

\section{CONCLUSION}

In this paper, we have given a unified relaxation framework for MIMO detection. The polynomial moment relaxation was developed including SDR as a special case. We have presented three approaches to replace the finite-alphabet constraint with a polynomial constraint. Since both the objective function and the constraints are polynomials after the transformation, we developed the moment relaxation approach by using the dual theories of moments and positive polynomials. The resulting problem can be solved by semi-definite programming with polynomial-time complexity. As the relaxation order increases, the solution of the relaxed problem monotonously converges to that of the MLD. Our PMR provides a flexible trade-off between complexity and performance. Interestingly, further work includes analyzing the probability of obtaining the ML solution with different relaxation order, the upper bound on the error probability, and the diversity order achieved by PMR.

\section{REFERENCES}

[1] G. D. Golden, G. J. Foschini, R. A. Valenzuela, and P. W. Wolniansky, "Detection algorithm and initial laboratory results using the V-BLAST space-time communication architecture," Electronics Letters, vol. 35, no. 1, pp. 14-15, Jan. 1999.

[2] E. Viterbo and J. Bouros, "A universal lattice code decoder for fading channels," IEEE Trans. Inform. Theory, vol. 45, no. 5, pp. 1639-1642, Jul. 1999.

[3] A. Burg, M. Borgmann, C. Simon, M. Wenk, M. Zellweger, and W. Fichtner, "Performance tradeoffs in the VLSI implementation of the sphere decoding algorithm," in Proc. IEE $3 G$ Mobile Communication Technologies Conference, London, UK, Oct. 2004.

[4] W.-K. Ma, T. Davidson, K. M. Wong, Z.-Q. Luo, and P.-C. Ching, "Quasi-maximum-likelihood multiuser detection using semi-definite relaxation with application to synchronous CDMA," IEEE Trans. Signal Processing, vol. 50, no. 4, pp. 912 - 922, April 2002.

[5] J. B. Lasserre, "Global optimization with polynomials and the problem of moments," SIAM J. Optim., vol. 11, no. 3, pp. 796-817, 2001.

[6] _ - "An explicit equivalent positive semidefinite program for nonlinear 0-1 programms," SIAM J. Optim., vol. 12, no. 3, pp. 756-769, 2002.

[7] L. Vandenberghe and S. Boyd, "Semidefinite programming," SIAM Review, vol. 38, pp. 49-95, 1996.

[8] B. Simon, "The classical moment problem as a self-adjoint finite difference operator," Adv. Math., vol. 137, pp. 82-203, 1998.

[9] J. F. Sturm, "Using SeDuMi 1.02, a MATLAB toolbox for optimization over symmetric cones," Optimization Methods and Software, vol. 11-12, pp. 625-653, 1999.

[10] R. Corless, P. Gianni, and B. Trager, "A reordered schur factorization method for zero-dimensional polynomial systems with multiple roots," in Proc. ACM Int. Symp. Symbolic and Algebraic Computation, Maui, Hawaii, 1997, pp. 133-140.

[11] C. Helmberg, F. Rendl, R. J. Vanderbei, and H. Wolkowicz, "An interior point method for semidefinite programming," SIAM J. Optim., vol. 6 , no. 2, pp. 342-361, May 1996. 